\title{
Impact of CT-scan on evaluation of tumor spread in patients with nasopharyngeal carcinoma.
}

\author{
Huynh Quang Huy* \\ Department of Radiology, Pham Ngoc Thach University of Medicine, Vietnam
}

\begin{abstract}
Background: Nasopharyngeal carcinoma, the most common neoplasm to arise in the nasopharynx, is a locally aggressive tumor with a high incidence of cervical nodal metastases. This study aimed to investigate the role of computed tomography in the evaluation of tumor stage ( $\mathrm{T}$ stage) and nodal stage ( $N$ stage) in patients with nasopharyngeal carcinoma.

Methods: CT scans of 338 newly diagnosed nasopharyngeal carcinoma patients were reviewed. All patients were restaged according to the 8th edition of the Union for International Cancer Control/ American Joint Committee on Cancer staging system relies on evaluation of the primary tumor ( $T$ category), the draining nodal groups ( $\mathrm{N}$ category).

Results: The 338 patients included in this analysis comprised 92 men and 246 women with a median age at diagnosis of 49 years (range, 10-89 years). WHO histopathologic type of non-keratinizing undifferentiated carcinoma was accounted $281 / 338(83.1 \%)$, non-keratinizing differentiated carcinoma was $15.1 \%$, and keratinizing squamous cell carcinoma was $1.8 \%$. With CT scan staging, the proportions of T1, T2, T3 and T4 were 8.9\% (30/338), $17.7 \%(60 / 338), 32.8 \%(111 / 338)$ and $40.5 \%(137 / 338)$, respectively; the proportions of N0, N1, N2 and N3 were $12.7 \%(43 / 338), 31.9 \%(108 / 338), 45.0 \%$ $(152 / 338)$, and $10.4 \%(35 / 338)$, respectively.

Conclusion: Computed tomography is essential for detection of early NPC, staging of the primary tumor, and evaluation of associated local lymphadenopathy.
\end{abstract}

Keywords: Computed tomography, Nasopharyngeal carcinoma, Tumor stage, Nodal stage.

Abbreviations: NPC: Nasopharyngeal Carcinoma; CT: Computed Tomography; HCMC: Ho Chi Minh City; NKUC: Non-Keratinizing Undifferentiated Carcinoma; NKDC: Non-Keratinizing Differentiated Carcinoma; KSCC: Keratinizing Squamous Cell Carcinoma; ${ }^{18}$ F-FDG. PET/CT: [18F] Fluoro-D-Glucose Positron-Emission Tomography.

Accepted on May 6, 2019

\section{Introduction}

Nasopharyngeal carcinoma (NPC), the most common neoplasm to arise in the nasopharynx, is a locally aggressive tumor with a high incidence of cervical nodal metastases. The tumor has a propensity towards extensive invasion into adjacent tissues, particularly laterally into the parapharyngeal space and superiorly into the skull base. However, spread to the palate, nasal cavity, and oropharynx have also commonly reported. Distant metastases can arise within bone, lung, the mediastinum and, more rarely, the liver [1-3]. Although NPC is rare in North America and Europe with an incidence of 0.5-2 per 100,000, intermediate incidence rates are seen in Southeast Asia, the Mediterranean Basin, and the Arctic ranging from 0.5 to 31.5 per 100,000 person-years in males and 0.1 to 11.8 person-years in females [4-6]. In southern China, NPC is endemic with overall NPC incidence rates reaching 20-30 per 100,000 person-years and $15-20$ per 100,000 person-years amongst males and females, respectively, in the province of Guangdong [7,8]. NPC has a male to-female ratio of 2-3:1 $[9,10]$, and is most common among patients 40-60 years old with bimodal age peaks in the second and sixth decades of life $[11,12]$.

Early detection, early diagnosis, accurate staging and evaluation after treatment had been the key to improve the efficacy of treatment and prolong survival period [13]. CT has been the most reliable and well-established imaging technique for staging and assessing the extent of nasopharyngeal carcinoma. The biggest advantage of CT imaging is that the surrounding bony destruction by NPC can be clearly visualized on CT image [13]. Zheng et al. [14] found that radiologists should pay attention to bony structures invaded by nasopharyngeal carcinoma on CT images. In addition, CT scan is very quick and gets abundant information. It is also 
relatively cheaper and still an effective imaging method for diagnosis and follow-up of nasopharyngeal carcinoma $[15,16]$.

This study aims to investigate the role of computed tomography $(\mathrm{CT})$ in the evaluation of tumor stage (T stage) and nodal stage ( $\mathrm{N}$ stage) in patients with nasopharyngeal carcinoma.

\section{Material and Methods}

From July 2015 to April 2019, a total of 338 consecutive patients with NPC visited HCMC Oncology Hospital, Vietnam. All patients with histologically proven NPC newly diagnosed were enrolled in this study. They completed a pre-treatment evaluation that included physical examination, nasopharyngeal fiberoptic endoscopy, CT scan of the nasopharynx and neck, chest radiography, abdominal sonography.

The CT scans were obtained with a GE Optima CT660, in axial plane and coronal, sagittal recontruction after the injection of contrast medium (Ultravist 370, Schering, Berlin, Germany) using $3 \mathrm{~mm}$ section thickness; $50 \mathrm{~mL}$ of Ultravist was administered by intravenous. All the images were reviewed and assessed by two of the authors independently. Cases with variable interpretation or disagreement in staging between the observers were reevaluated side by side, and the differences were confirmed to reach a final consensus.

Medical records and imaging studies were analyzed, and all patients were restaged according to the 8th edition of the Union for International Cancer Control/American Joint Committee on Cancer staging system [17] relies on evaluation of the primary tumor ( $\mathrm{T}$ category), the draining nodal groups ( $\mathrm{N}$ category). Histopathologic evaluation was according to the 2003 World Health Organization classification: non-keratinizing undifferentiated carcinoma (NKUC), non-keratinizing differentiated carcinoma (NKDC), keratinizing squamous cell carcinoma (KSCC).

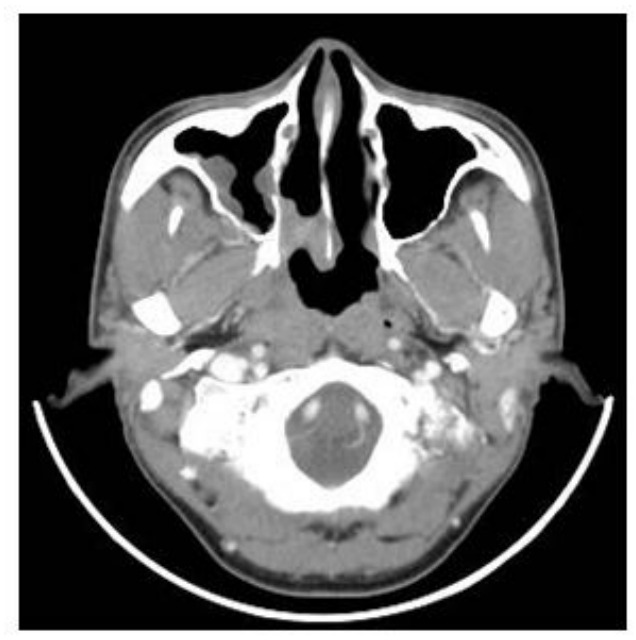

(a)
SPSS version 20.0 (IBM, Armonk, NY) was used for all statistical analyses.

\section{Results}

The 338 patients included in this analysis comprised 92 men and 246 women with a median age at diagnosis of 49 years (range, 10-89 years). WHO histopathologic type of NKUC was acounted $281 / 338$ (83.1\%), NKDC was $15.1 \%$, KSCC was $1.8 \%$. According to the eighth edition UICC/AJCC staging standards, the proportions of T1, T2, T3 and T4 were $8.9 \%$ $(30 / 338), \quad 17.7 \%(60 / 338), \quad 32.8 \% \quad(111 / 338)$ and $40.5 \%$ (137/338), respectively; the proportions of N0, N1, N2 and N3 were $12.7 \%$ (43/338), 31.9\% (108/338), 45.0\% (152/338), and $10.4 \%(35 / 338)$ respectively (Table 1$)$. Figures $1-4$ are the enhanced CT images of patients in the study.

Table 1. Clinical and histopathologic characteristics of the study patients.

\begin{tabular}{lcl}
\hline Variables & Number & $\%$ \\
\hline$<50$ & Age (year) & \\
\hline$\geq 50$ & 176 & 52.1 \\
\hline & 162 & 47.9 \\
\hline Male & \multicolumn{2}{c}{ Sex } \\
\hline Female & 92 & 27.2 \\
\hline & 246 & 72.8 \\
\hline NKUC & WHO histopathologic type & \\
\hline NKDC & 281 & 83.1 \\
\hline KSCC & 51 & 15.1 \\
\hline
\end{tabular}

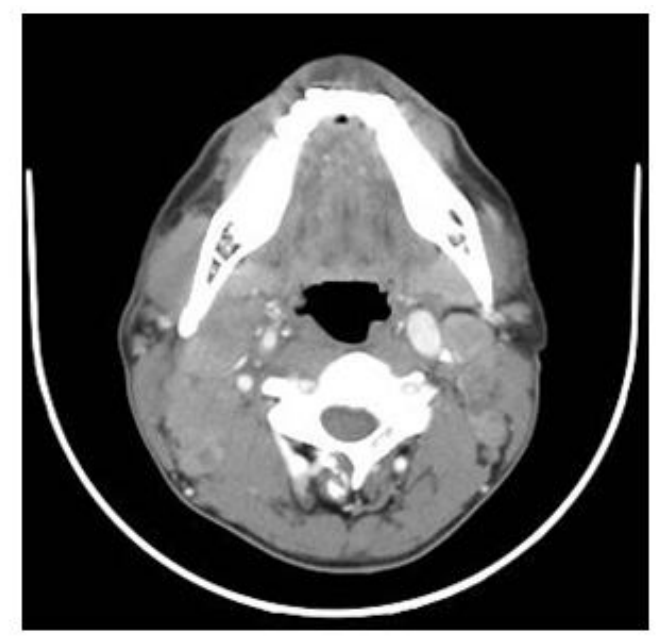

(b)

Figure 1. Enhanced CT image: (a) Nasopharyngeal carcinoma with posterior nasal cavity and right cavernous sinus infiltration; (b) both side lymph node metastasis at level II, III, IV. 


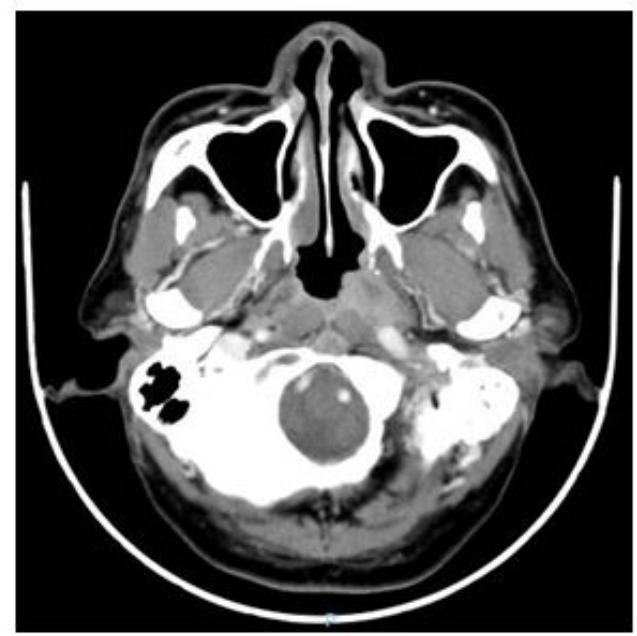

(a)

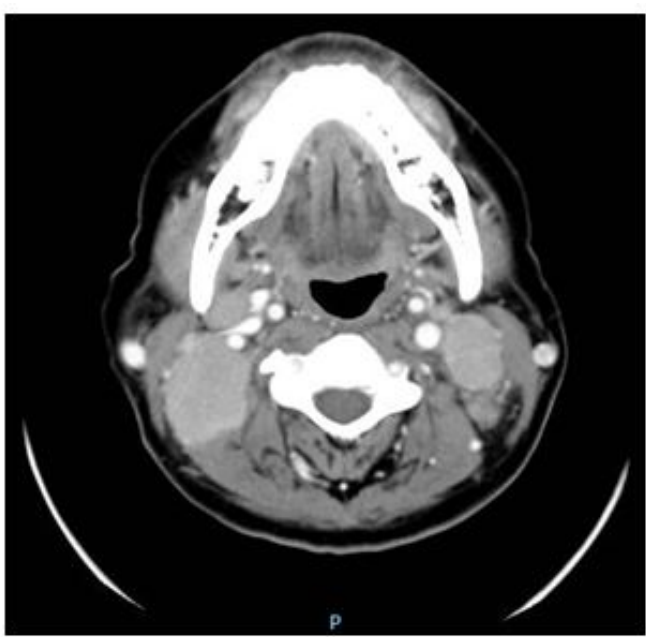

(b)

Figure 2. Enhanced CT image of tumor invasion into the sphenoid sinus and bone destruction in patient with NPC (a); and both side lymph node metastasis at level II, III (b).

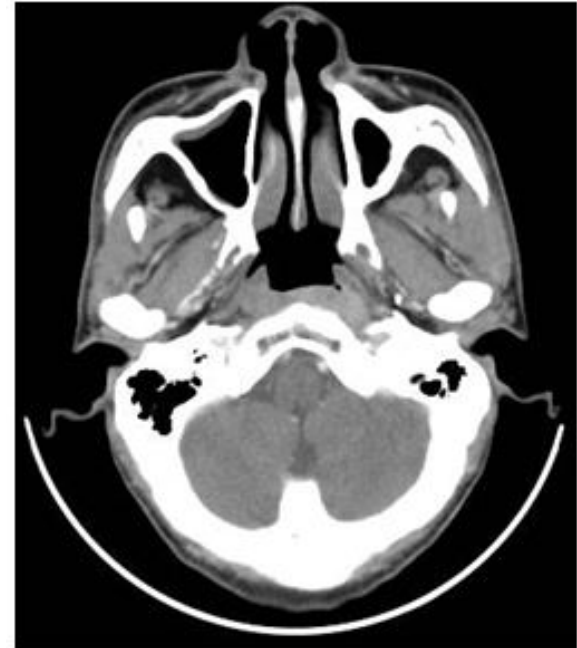

(a)

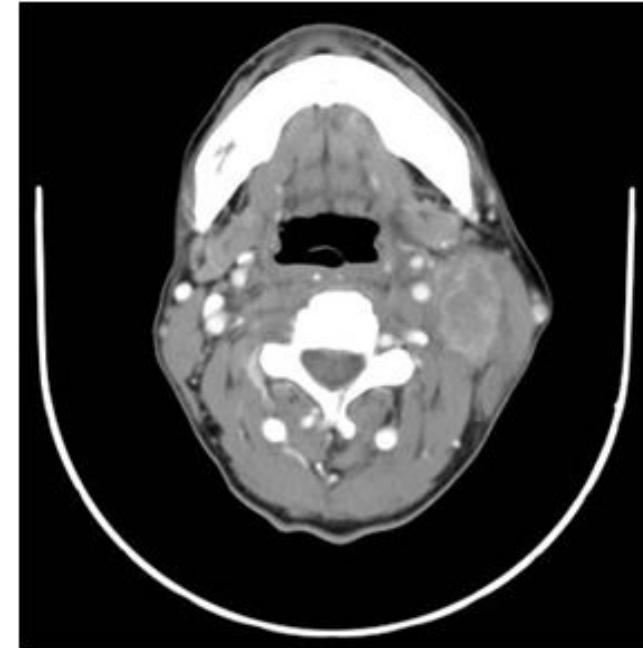

(b)

Figure 3. (a) Enhanced CT image of NPC and (b) Lymph node metastasis at level II, III with inside necrosis.

\section{Discussion}

Nasopharyngeal carcinoma is a unique disease with clinical behavior, epidemiology, and histopathology that is different from that of squamous cell carcinomas of the head and neck. NPC accounts for $0.25 \%$ of all malignancies in the United States and $15-18 \%$ of malignancies in southern China. It also accounts for $10-20 \%$ of childhood malignancies in Africa. The male to-female ratio is $3: 1$. It is the most common among patients 40-60 years old, and bimodal age peaks occur in the second and sixth decades of life [18-22]. NPC is caused by the interaction of genetic susceptibility, environmental factors (e.g., exposure to chemical carcinogens), and infection with Epstein-Barr virus. High antibody titers to Epstein-Barr virus antigens are useful diagnostic markers, and there are many tests to detect both IgG and IgA titers. In China, dietary factors for NPC include nitrosamine-rich salted food [19-22]. Patients often present with local symptoms, such as epistaxis and a blocked nose, but may also present with hearing loss, otalgia, headache, or cranial nerve involvement. However, the nasopharynx is a relatively clinically silent area. The first presentation may be with cervical nodal or distant metastasis [19-23]. Our results of patient demographics were consistent with the findings of previous researches with the median age at diagnosis of 49 years (range 10-89 years) and male/femal ratio was $1 / 2.67$. 


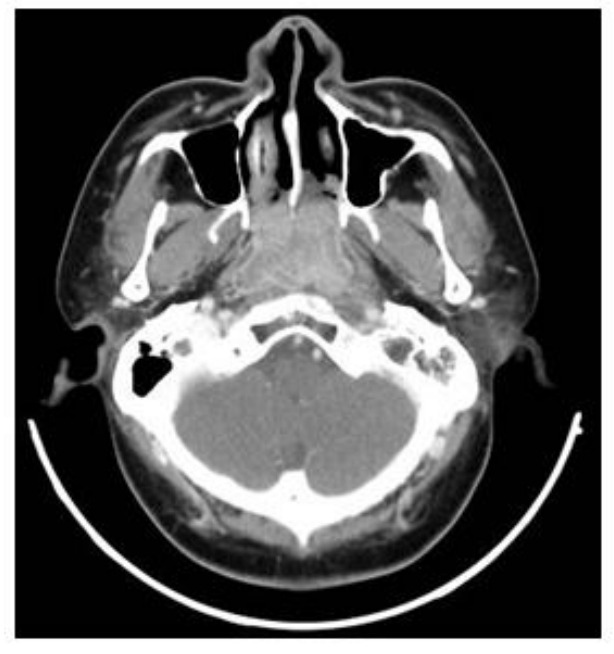

(a)

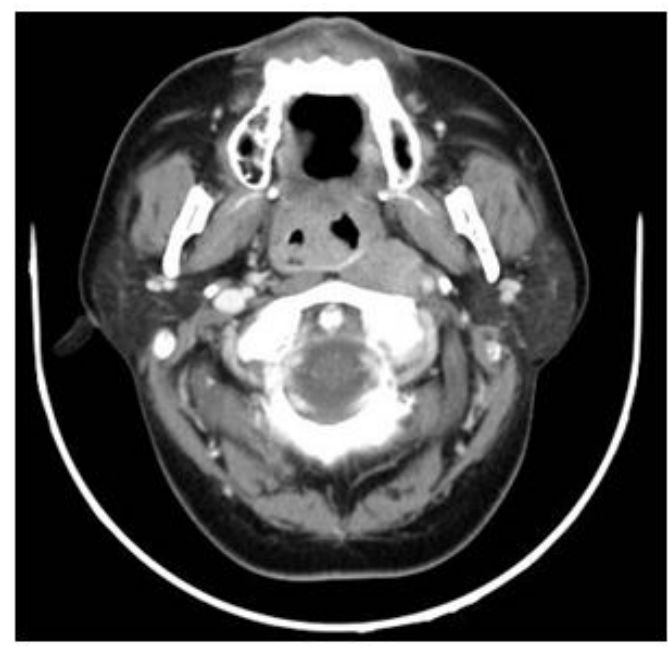

(b)

Figure 4. Enhanced CT image of NPC: (a) Fossa of Rosenmuller was narrowed due to the tumor invasion; (b) Retropharyngeal lymph node metastases.

The World Health Organization classification of NPC recognizes three histologic types. Keratinizing squamous cell carcinoma (type 1) is found more often in nonendemic areas and has the worst prognosis. It is analogous to squamous cell carcinoma elsewhere in the pharynx and is associated with cigarette and alcohol use. Nonkeratinizing carcinoma (type 2) behaves in a fashion similar to type 3 . Both types are radiosensitive and have a much better prognosis. Undifferentiated carcinoma (type 3 ) was previously called B lymphoepithelioma because of the mix of undifferentiated epithelial and nonmalignant $\mathrm{T}$ lymphocytes. In North America, around $25 \%$ of patients with NPC have type $1,12 \%$ have type 2 , and $63 \%$ have type 3 . The histologic distribution in southern China is $2 \%, 3 \%$, and $95 \%$, respectively [20-23]. In our study, NKUC was counted for $281 / 338$ (83.1\%), NKDC was $15.1 \%$, KSCC was $1.8 \%$.

CT has long been used for staging NPC, especially for the detection of skull base tumor involvement with lytic or sclerotic lesions [23,24], but it has now largely been replaced by MRI for primary and nodal staging. However, CT is still used for radiotherapy planning and, in some centers, is used together with PET using ${ }^{18} \mathrm{~F}-\mathrm{FDG}$. PET/CT has been shown to be of value in NPC staging, where the main advantage is for the detection of distant metastasis [25]. It is also used for monitoring patients after therapy and detecting NPC recurrence.

Staging of NPC according to the seventh edition of the American Joint Committee on Cancer's TNM staging system [17] relies on evaluation of the primary tumor (T category), the draining nodal groups ( $\mathrm{N}$ category) and evidence or absence of metastatic disease (M category).

\section{Category T1 NPC}

Tumor confined to the nasopharynx was found in only 30/338 of patients $(8.9 \%)$ (Table 2). Mucosal spread of NPC tends to involve the superior portion of the nasopharynx. Deep infiltrating tumors may be found even when the nasopharyngeal component is small $[18,26]$.

Table 2. $T$ stage and $N$ stage.

\begin{tabular}{lll}
\hline Variables & Number & $\%$ \\
\hline T1 & T stage & \\
\hline T2 & 30 & 8.9 \\
\hline T3 & 60 & 17.7 \\
\hline T4 & 111 & 32.8 \\
\hline & 137 & 40.5 \\
\hline N0 & N stage & \\
\hline N1 & 43 & 12.7 \\
\hline N2 & 108 & 31.9 \\
\hline N3 & 152 & 45 \\
\hline
\end{tabular}

The nasal cavity is commonly involved by NPC. Minimal invasion of tumor to the margin of the choanal orifice is common, whereas more bulky disease extending into the main body of the nasal cavity is encountered less frequently. NPC at the roof may spread centrally along the septum [20,26].

Inferior superficial extension down to the mucosa of the oropharynx is uncommon. Invasion of the oropharynx rarely occurs as an isolated event and therefore is not usually an early sign of disease $[18,26]$.

\section{Category T2 NPC}

Parapharyngeal spread occurs when tumor spreads posterolaterally and usually involves lateral penetration 
through the levator palatini muscle and pharyngobasilar fascia to involve the tensor palatini muscle and parapharyngeal fat space. Invasion of the parapharyngeal space is associated with an increased risk of distant metastases and tumor recurrence. It can lead to compression of the eustachian tube with middle ear and mastoid effusion. Further posterolateral spread may also involve the carotid space and encase the carotid artery [25].

Retropharyngeal spread occurs when tumor spreads posteriorly to involve longus capitis muscles and prevertebral space. This region contains lymphatics and a venous plexus, and so invasion of the prevertebral space is associated with an increased risk of distant metastases. In some patients, this posterior extension is the preferred pattern of tumor spread, with bulky disease continuing down to the foramen magnum and upper cervical spine [27].

\section{Category T3 NPC}

NPC has a propensity to invade the skull base at diagnosis. The clivus, pterygoid bones, body of the sphenoid, and apices of the petrous temporal bones are most commonly invaded. Axial T1-weighted imaging provides a good overview of the extent of skull base invasion [18,20]. CT reveals permeative or erosive bone changes of the skull base or spread along foraminal pathways. Also, sclerosis of the pterygoid process with increased attenuation of medullary cavity or thickening of cortical bone may be detected [28]. Tumor frequently invades the skull base foramina (foramen rotundum, oval, and lacerum and vidian canal) and fissures (pterygomaxillary and petroclival). Tumor extended into the pterygopalatine fossa provides a route of spread to the orbit, infratemporal fossa, nasal cavity, and middle cranial fossa. Invasion of hypoglossal nerve canal and jugular foramen is less common [18,29].

Paranasal sinus involvement occurs as a result of direct extension. Maxillary sinus involvement occurs after nasal or infratemporal maxillary wall erosion (6\%). Sphenoid sinus extension is common because it lies above the roof of the nasopharynx. The ethmoid and sphenoid are less commonly involved. Sinus involvement is recognized by the loss of contiguity of the sinus walls. Intrasinus extension of tumor may be seen. Tumor can be differentiated from reactive mucosal thickening on MRI, where inflammatory mucosal thickening is seen as uniform T2-weighted signal greater than that of tumor, also enhancing to a greater degree than tumor $[18,26]$.

\section{Category T4 NPC}

Meningeal involvement appears as nodular enhancement, often along the floor of middle cranial fossa or posterior to the clivus. Direct invasion of the brain is rare. Invasion of cavernous sinus can lead to multiple cranial palsies. NPC may spread into the cavernous sinus from tumor surrounding the horizontal portion of the internal carotid artery, foramen ovale, orbital fissures, or directly through the skull base $[18,23,26]$. NPC has a propensity to spread to nodes and in $87.3 \%$ (Table 1 ) is found by imaging to have a tendency for bilateral neck spread. Nodal metastases are diagnosed if the shortest nodal axial diameter reaches $5 \mathrm{~mm}$ or greater in the lateral retropharyngeal region, $11 \mathrm{~mm}$ in the jugulodigastric region, or $10 \mathrm{~mm}$ in other non-retropharyngeal nodes of the neck; if there is a group of three or more nodes that are borderline in size; or if the nodes display necrosis or extracapsular spread. Extracapsular spread has also been shown to be an independent prognostic factor [25,30].

The diagnosis of enlarged retropharyngeal lymph nodes in patients with NPC can only be made by imaging and MRI has an advantage over $\mathrm{CT}$ in being better able to separate the lateral retropharyngeal nodes from the primary tumor in the adjacent posterolateral nasopharynx. Lateral retropharyngeal nodes are among the most common sites of nodal spread from NPC and have been considered the first echelon of metastatic spread [26]. However, nodal spread may bypass these nodes and spread to other nodes of the upper neck. Metastatic lateral retropharyngeal nodes can be identified from the skull base to the level of $\mathrm{C} 3$. Retropharyngeal node involvement is now classified as category $\mathrm{N} 1$, whether unilateral or bilateral [18,31]. PET/CT reveals increased FDG uptake in metastatic cervical lymph nodes, but MRI appears to be superior to $\mathrm{PET} / \mathrm{CT}$ for the assessment of retropharyngeal nodal metastasis because of the better discrimination of nodes from the adjacent primary tumor [32].

Metastatic nodes posterior to the jugular vein in the upper neck are the most common sites for nonretropharyngeal nodes [30] and are designated as high internal jugular nodes, although at this site, the internal jugular and spinal accessory nodal chains converge. Nodes then usually spread in an orderly sequence down the neck. Nodes in the submandibular and parotid or periparotid region are far less common at diagnosis. Nodal metastases at supraclavicular fossa increase the incidence of distant metastases [18].

NPC shows a high frequency of distant metastases (5-41\%). The most common sites of metastases include bone $(20 \%)$, lung (13\%), and liver (9\%). Patients with supraclavicular lymphadenopathy or tumors extension into the parapharyngeal and retropharyngeal space have a significantly higher risk of distant metastases. PET/CT is sensitive to detect bony and softtissue metastatic deposits [25]. Whole-body MRI shows a diagnostic capacity similar to that of FDG PET/CT in assessing distant-site status in patients with untreated NPC; in one reported study, the combined interpretation of whole-body MRI and FDG PET/CT showed no significant benefit over either technique alone [32]. The frequency of distant metastases in our study was $19.2 \%$ which was consistent with previous reports. Our data did not contain distant metastases because of CT only scan neck area with or without head area. This is one of the limitations of our study.

\section{Conclusion}

In conclusion, computed tomography is essential for detection of early NPC, staging of the primary tumor, and evaluation of associated local lymphadenopathy. Further studies are needed 
to detect tumor recurrence and radiation-associated changes in the soft tissue and bone after therapy.

\section{Conflicts of Interest}

The authors declare no conflicts of interest.

\section{Ethics Approval and Consent to Participate}

The present study was approved by the HCMC Oncology Hospital Ethical Committee. Informed consent was waived. Consent for publication is not applicable.

\section{References}

1. Adham M, Kurniawan AN, Muhtadi AI, Roezin A, Hermani B, Gondhowiardjo S, Tan IB, Middeldorp JM. Nasopharyngeal carcinoma in Indonesia: epidemiology, incidence, signs, and symptoms at presentation. Chin J Cancer 2012; 31:185-196.

2. Haleshappa RA, Thanky AH, Kuntegowdanahalli L, Kanakasetty GB, Dasappa L, Jacob L. Epidemiology and outcomes of nasopharyngeal carcinoma: experience from a regional cancer center in Southern India. South Asian J Cancer 2017; 6:122-124.

3. Yu MC, Yuan JM. Epidemiology of nasopharyngeal carcinoma. Semin Cancer Biol 2002; 12:421-429.

4. Chang ET, Adami HO. The enigmatic epidemiology of nasopharyngeal carcinoma. Cancer Epidemiol Biomarkers Prev 2006; 15:1765-1777.

5. Huang SJ, Tang YY, Liu HM, Tan GX, Wang X, Zhang H, Yang F, Yang S. Impact of age on survival of locoregional nasopharyngeal carcinoma: An analysis of the surveillance, epidemiology, and end results program database, 2004-2013. Clin Otolaryngol 2018; 43:1209-1218.

6. Lv JW, Huang XD, Chen YP, Zhou GQ, Tang LL, Mao YP, Li WF, Lin AH, Ma J, Sun Y. A national study of survival trends and conditional survival in nasopharyngeal carcinoma: analysis of the national population-based surveillance epidemiology and end results registry. Cancer Res Treat 2018; 50:324-334.

7. Friborg J, Wohlfahrt J, Melbye M. Familial risk and clustering of nasopharyngeal carcinoma in Guangdong, China. Cancer 2005; 103: 211.

8. He YQ, Xue WQ, Shen GP, Tang LL, Zeng YX, Jia WH. Household inhalants exposure and nasopharyngeal carcinoma risk: a large-scale case-control study in Guangdong, China. BMC Cancer 2015; 15:1022.

9. OuYang PY, Zhang LN, Lan XW, Xie C, Zhang WW, Wang QX, Su Z, Tang J, Xie FY. The significant survival advantage of female sex in nasopharyngeal carcinoma: a propensity-matched analysis. $\mathrm{Br}$ J Cancer 2015; 112:1554-1561.

10. Xie SH, Yu IT, Tse LA, Mang OW, and Yue L. Sex difference in the incidence of nasopharyngeal carcinoma in Hong Kong 1983-2008: suggestion of a potential protective role of oestrogen. Eur J Cancer 2013; 49:150-155.
11. Wu SG, Liao XL, He ZY, Tang LY, Chen XT, Wang Y, Lin Q. Demographic and clinicopathological characteristics of nasopharyngeal carcinoma and survival outcomes according to age at diagnosis: A population-based analysis. Oral Oncol 2017; 73:83-87.

12. Xiao G, Cao Y, Qiu X, Wang W, Wang Y. Influence of gender and age on the survival of patients with nasopharyngeal carcinoma. BMC Cancer 2013; 13:226.

13. Chung NN, Ting LL, Hsu WC, Lui LT, Wang PM. Impact of magnetic resonance imaging versus $\mathrm{CT}$ on nasopharyngeal carcinoma: primary tumor target delineation for radiotherapy. Head Neck 2004; 26: 241-246.

14. Zheng GL, Zeng QX, Wu PH, Yuan CM. Computed tomography in the management of nasopharyngeal carcinoma. Clin Radiol 1989; 40:25-29.

15. Ma X, Lu JJ, Loh KS, Shakespeare TP, Thiagarajan A, Goh $\mathrm{BC}$, and Tan KS. Role of computed tomography imaging in predicting response of nasopharyngeal carcinoma to definitive radiation therapy. Laryngoscope 2006; 116:2162-2165.

16. Su D, Jin G, Xie D, Liu Y. Identification of local recurrence and radiofibrosis by computed tomography perfusion on nasopharyngeal carcinoma after radiotherapy. Can Assoc Radiol J 2010; 61: 265-270.

17. Doescher J, Veit JA, Hoffmann TK. The 8th edition of the AJCC cancer staging manual : updates in otorhinolaryngology, head and neck surgery. HNO 2017; 65:956-961.

18. King AD, Bhatia KS. Magnetic resonance imaging staging of nasopharyngeal carcinoma in the head and neck. World J Radiol 2010; 2: 159-165.

19. Chong VF, Ong CK. Nasopharyngeal carcinoma. Eur J Radiol 2008; 66:437-447.

20. Glastonbury CM. Nasopharyngeal carcinoma: the role of magnetic resonance imaging in diagnosis, staging, treatment, and follow-up. Top Magn Reson Imaging 2007; 18: 225-235.

21. Dubrulle F, Souillard R, Hermans R. Extension patterns of nasopharyngeal carcinoma. Eur Radiol 2007; 17:2622-2630.

22. Chin SC, Fatterpekar G, Chen CY, Som PM. MR imaging of diverse manifestations of nasopharyngeal carcinomas. AJR Am J Roentgenol 2003; 180: 1715-1722.

23. Weber AL, al-Arayedh S, Rashid A. Nasopharynx: clinical, pathologic, and radiologic assessment. Neuroimaging Clin N Am 2003; 13:465-483.

24. Goh J, Lim K. Imaging of nasopharyngeal carcinoma. Ann Acad Med Singapore 2009; 38:809-816.

25. Ng SH, Chan SC, Yen TC, Chang JT, Liao CT, Ko SF, Wang HM, Wai YY, Wang JJ, Chen MC. Pretreatment evaluation of distant-site status in patients with nasopharyngeal carcinoma: accuracy of whole-body MRI at 3-Tesla and FDG-PET-CT. Eur Radiol 2009; 19:2965-2976.

26. King AD, Lam WW, Leung SF, Chan YL, Teo P, Metreweli C. MRI of local disease in nasopharyngeal carcinoma: 
tumour extent vs tumour stage. Br J Radiol 1999; 72:734-741.

27. Lee CC, Chu ST, Chou P, Lee CC, Chen LF. The prognostic influence of prevertebral space involvement in nasopharyngeal carcinoma. Clin Otolaryngol 2008; 33:442-449.

28. Chen L, Liu LZ, Mao YP, Tang LL, Sun Y, Chen Y, Lin AH, Li L, Ma J. Grading of MRI-detected skull-base invasion in nasopharyngeal carcinoma and its prognostic value. Head Neck 2011; 33:1309-1314.

29. Shatzkes DR, Meltzer DE, Lee JA, Babb JS, Sanfilippo NJ, Holliday RA. Sclerosis of the pterygoid process in untreated patients with nasopharyngeal carcinoma. Radiology 2006; 239: 181-186.

30. Wang XS, Hu CS, Ying HM, Zhou ZR, Ding JH, Feng Y. Patterns of retropharyngeal node metastasis in nasopharyngeal carcinoma. Int J Radiat Oncol Biol Phys 2009; 73: 194-201.
31. Tang L, Li L, Mao Y, Liu L, Liang S, Chen Y, Sun Y, Liao X, Tian L, Lin A, Liu M, Ma J. Retropharyngeal lymph node metastasis in nasopharyngeal carcinoma detected by magnetic resonance imaging : prognostic value and staging categories. Cancer 2008; 113: 347-354.

32. King AD, Ma BB, Yau YY, Zee B, Leung SF, Wong JK, Kam MK, Ahuja AT, Chan AT. The impact of 18F-FDG PET/CT on assessment of nasopharyngeal carcinoma at diagnosis. Br J Radiol 2008; 81:291-298.

\section{*Correspondence to}

Huynh Quang Huy

Department of Radiology

Pham Ngoc Thach University of Medicine

Vietnam 